\title{
Producción orgánica de Beta vulgaris subespecie cicla con inoculantes microbianos
}

\author{
Organic production of Beta vulgaris subespecie cicla with microbial inoculants
}

\author{
José Venegas-González, Carlos Méndez-Inocencio, Erika Karina Martínez-Mendoza, Luis Fernando Ceja Torres y \\ María Dolores Rodríguez-Torres* \\ Instituto Politécnico Nacional, CIIDIR Unidad Michoacán. Justo Sierra No. 28, C. P. 59510, Jiquilpan Michoacán, México.
}

\section{RESUMEN}

Los sustratos orgánicos son adecuados para la producción de hortalizas; además, al asociarlos con inoculantes microbianos favorecen su aprovechamiento. El objetivo del estudio fue evaluar el efecto de microorganismos benéficos conjuntamente con sustratos orgánicos sobre la nutrición de la acelga. Dos cepas de Bacillus subtilis y una de Trichoderma harzianum, y su combinación con el sustrato orgánico se evaluaron para promover el crecimiento de la hortaliza. Se evaluó la producción de biomasa fresca y seca y los elementos esenciales. La biomasa fresca no mostró diferencias significativas entre tratamientos en el primer corte. La cepa C90_3T de B. subtilis tuvo la máxima producción de biomasa fresca en el segundo corte. La combinación de ambas cepas de Bacillus propició la absorción de varios elementos esenciales. Los microorganismos evaluados incrementaron la absorción nutrimental, el crecimiento y la producción de las plantas desarrolladas en el sustrato.

Palabras clave: microorganismos benéficos, sustratos, promoción de crecimiento, acelga, agricultura sustentable

\section{ABSTRACT}

Organic substrates are suitable for vegetable production; in addition, by associating them with microbial inoculants, they favor their use. The objective of this study was to evaluate the effect of beneficial microorganisms, together with organic substrates, on the nutrition of Swiss chard. Two Bacillus subtilis and one Trichoderma harzianum strains, and their combination with the organic substrate, were evaluated to promote the vegetable growth. Fresh and dry biomass production and the essential elements were evaluated. Fresh biomass did not show significant differences among treatments in the first cut. Strain C90_3T of B. subtilis had the highest production of fresh biomass in the second cut. The combination of both Bacillus strains led to the absorption of several essential elements. The microorganisms evaluated increased the nutrimental absorption, growth and production of plants developed in the substrate.

Key words: beneficial microorganisms, substrates, growth promotion, Swiss chard, sustainable agriculture

\section{INTRODUCCIÓN}

La explotación agrícola y ganadera y la industrialización de sus productos son fuente de materiales contaminan- tes de agua, suelo, atmósfera, cultivos, animales y humanos (Pinos-Rodríguez et al., 2012). Los residuos sólidos orgánicos domésticos e industriales pueden usarse para producir compost, sustratos y abonos orgánicos para la producción de hortalizas (Pan et al., 2012). Esto contribuye a la disminución de la contaminación ambiental por reducción de la emisión de gases con efecto invernadero, como el $\mathrm{CO}_{2^{\prime}} \mathrm{CH}_{4}$ y $\mathrm{N}_{2} \mathrm{O}$ (Boldrin et al., 2009). A la vez, estos gases contribuyen al calentamiento global y al cambio climático (Chu et al., 2004; Boldrin et al., 2009). Un reto que se tiene a este momento es el uso eficiente de los fertilizantes para disminuir las pérdidas y los efectos negativos al medioambiente (Adesemoye y Kloepper, 2009).

El interés por la búsqueda de enmiendas orgánicas e inoculantes biológicos que favorezcan la disponibilidad de los nutrimentos necesarios para el cultivo y que puedan disminuir el uso de fertilizantes, e incluso evitarlo, en producción de autoconsumo y huertos orgánicos ha ido en aumento (Abedini Aboksari et al., 2018). La inoculación de microorganismos promotores del desarrollo vegetal a los cultivos de interés agrícola por su potencial como biofertilizantes es promisorio, por su habilidad de estimular el crecimiento de las plantas a través de diferentes mecanismos directos e indirectos, por ejemplo, la estimulación del crecimiento de la raíz (producción de fitohormonas), rizoremediación, el control del estrés de la planta, la antibiosis, inducción de resistencia sistémica y antagonismo de patógenos a través de la competencia por nutrientes y nichos (Lugtenberg y Kamilova, 2009). Tanto hongos como bacterias se evalúan por su alto potencial como inoculantes biológicos para el desarrollo de las plantas, por ejemplo, Bacillus subtilis son bacterias promotoras del desarrollo vegetal, solubilizadoras de fósforo y antagónicas a patógenos, en ese mismo sentido, Trichoderma harzianum tiene potencial como promotor del desarrollo de plantas, protector contra fitopatógenos e incluso ayudando a tolerar el estrés salino (Pérez-García et al., 2011; Kleifeld y Chet, 1992). El desarrollo de inoculantes eficientes depende de las interacciones sustrato-planta-microorganismo; es decir, los exudados de la planta, el tipo de suelo, la humedad, las perturbaciones causadas por las prácticas de manejo, la concentración de nutrientes o la diversidad microbiana son sólo algunos factores que intervienen en la eficacia de un microorganismo promotor de crecimiento (Rivera-Cruz et al., 2008; Souza et al., 2015). 
La acelga (Beta vulgaris subsp cicla Ford Hook Giant) es una planta bienal herbácea, excelente para la dieta humana por su alto contenido de vitamina A, C, B, calcio, hierro, fósforo, compuestos fenólicos y actividad antioxidante (Pyo et al., 2004; Anthony et al., 1992). La acelga es un cultivo con una alta demanda en el mercado por sus hojas y es común en producción intensiva por métodos convencionales con uso de fertilizantes, y aunque hay pocos reportes, el rendimiento disminuye cuando su producción es orgánica (Lampkin, 1990) o no se presentan diferencias con el uso de biofertilizantes (Medeiros et al., 2008). Sin embargo, esto puede ser corregido con el uso de inoculantes microbianos y con el uso de fuentes con contenidos elevados de nitrógeno, para la preparación de sustratos orgánicos.

El objetivo del presente estudio fue evaluar un sustrato orgánico solo y con la inoculación de $B$. subtilis y $T$. harzianum únicos y su combinación que coadyuven al uso eficiente del sustrato para la nutrición y producción de acelga sin la aplicación de fertilizantes sintéticos.

\section{MATERIALES Y MÉTODOS}

Compostaje de materiales para la obtención del sustrato

La composición del sustrato orgánico consistió de una mezcla de cascarilla de arroz, polvo de fibra de coco, harina de pescado, cachaza de caña de azúcar, estiércol de bovino, roca fosfórica y la dolomita, además, durante el proceso de compostaje se utilizó levadura de cerveza (Tabla 1). El sustrato lo obtuvimos al mezclar los materiales y humedecerlos hasta $60 \%$ de capacidad de retención de agua, revolviendo la mezcla cada tercer día. A los 60 días el sustrato se dejó secar al ambiente y se realizaron análisis químicos y fisicoquímicos, pH (relación agua:sustrato 2:1), conductividad eléctrica (CE), Nitrógeno total $(\mathrm{N})$ (microKjeldahl), fósforo asimilable $(\mathrm{P})$ (Olsen y Sommers, 1982) y la determinación por espectrofotometría de absorción atómica de K (Potasio), Calcio (Ca), Magnesio (Mg), Hierro (Fe), Cobre (Cu), Manganeso (Mn) y Zinc (Zn).

\section{Microorganismos estudiados}

Las cepas utilizadas para evaluar su potencial como promotoras de crecimiento fueron: $B$. subtilis CH90_3T (proporcionada del cepario de Laboratorio de Bacteriología Molecular de la Dra. Gabriel Olmedo del Centro de Investiga-

Tabla 1. Materiales que constituyen al sustrato y sus proporciones. Table 1. Materials that constitute the substrate and its proportions.

\begin{tabular}{lc}
\hline Componente & Proporción (\%) \\
\hline Polvo de fibra de coco (PFC) & 50.0 \\
\hline Estiércol bovino (CEB) & 15.8 \\
\hline Cascarilla de arroz (CA) & 10.0 \\
\hline Harina de pescado (HP) & 10.0 \\
\hline Cachaza de caña de azúcar (CCCA) & 10.0 \\
\hline Dolomita (DO) & 1.0 \\
Roca fosfórica (RF) & 3.0 \\
Levadura de cerveza (LV) & 0.2 \\
\hline
\end{tabular}

ción y de Estudios Avanzados; CINVESTAV Unidad Irapuato), B. subtilis cepa A1 y T. harzianum cepa Th_C2 (fueron obtenidas del cepario del laboratorio de fitopatología del Centro Interdisciplinario de Investigación para el Desarrollo Integral Regional; CIIDIR Unidad Michoacán). Para la preparación del inóculo, los microorganismos fueron mantenidos en medio papa dextrosa agar (PDA) y se incubaron a $27^{\circ} \mathrm{C}$ durante $24 \mathrm{~h}$.

\section{Efecto de la inoculación de microorganismos sobre la promoción de crecimiento en plantas de acelga}

Para evaluar el rendimiento de acelga sobre sustrato orgánico y el efecto de los microorganismos para la promoción de crecimiento. Primero se germinaron las semillas de acelga en peat moss; una vez germinadas, los microorganismos fueron inoculados sobre la raíz por inmersión dependiendo del tratamiento, sólo o en combinación a una densidad de 1 x $10^{8} \mathrm{UFC} / \mathrm{ml}$ (Unidades Formadoras de Colonias). Se empleó un diseño completamente aleatorizado con ocho tratamientos (Testigo, CH90_3T, A1, Th_C2, CH90_3T $+\mathrm{A} 1, \mathrm{CH} 90 \_3 \mathrm{~T}+\mathrm{Th} \_\mathrm{C} 2, \mathrm{~A} 1+\mathrm{Th}$ C2, $\mathrm{A} 1+\mathrm{CH} 90 \_3 \mathrm{~T}+\mathrm{Th}$ C2) y 10 repeticiones. Cinco plántulas se trasplantaron en contenedores de $10 \mathrm{~L}$ con el sustrato antes mencionado bajo condiciones de invernadero.

\section{Evaluación de parámetros en plantas de acelga}

Durante el experimento se tomaron datos de biomasa de 3 cosechas de hojas a los 60, 75 y 90 días después del trasplante, registrando datos de biomasa fresca y seca. Para determinar la biomasa seca, se tomó una muestra compuesta por tratamientos, que constó de una hoja por repetición, las cuales se secaron a $60^{\circ} \mathrm{C}$ en estufa hasta peso constante. El peso obtenido se usó para calcular el peso seco total por repetición. Además, a la muestra utilizada para el cálculo de la biomasa seca de cada tratamiento se utilizó como muestra compuesta para el análisis foliar de la acelga determinando $\mathrm{N}, \mathrm{P}, \mathrm{K}, \mathrm{Ca}, \mathrm{Mg}, \mathrm{Fe}, \mathrm{Cu}, \mathrm{Mn}$ y Zn.

\section{Análisis estadístico}

Con los datos de rendimiento fresco y seco, se corrió un análisis de varianza de una vía, y se realizó una prueba de comparación de medias empleando la prueba de Tukey ( $p \leq 0.05$ ) para lo cual se empleó el programa SAS 9.0 (SAS Institute Inc. 2012).

\section{RESULTADOS Y DISCUSIÓN}

Variables químicas y fisicoquímicas del sustrato orgánico

Los análisis fisicoquímicos del sustrato orgánico, indican que la mezcla de los materiales empleados, produce un sustrato con características favorables desde el punto de vista nutricional para el cultivo de hortalizas (Tabla 2). El contenido de macronutrimentos (N, P, K, Ca, y Mg) es alto, así como el de micronutrimentos, excepto el $\mathrm{Cu}$ el cual es bajo en el sustrato, pudiendo estar como trazas, pues de acuerdo al método utilizado en la muestra no fue detectado.

Entre las características fisicoquímicas, el pH y el contenido de sales son altos ( $\mathrm{pH}=8.5$ y $\mathrm{CE}=5070 \mu \mathrm{S} / \mathrm{cm}$ ), lo que 
Tabla 2. Análisis químico y fisicoquímico del sustrato.

Table 2. Substrate chemical and physicochemical analysis.

\begin{tabular}{|c|c|c|c|c|c|}
\hline & \multicolumn{4}{|c|}{ Repeticiones } & \multirow[t]{2}{*}{ Desv. Std. } \\
\hline & I & II & III & Media & \\
\hline pH & 8.4 & 8.1 & 9.0 & 8.5 & 0.41 \\
\hline EC & 4980 & 5115 & 5115 & 5070 & 63.64 \\
\hline N \% & 3.15 & 2.48 & 1.87 & 2.5 & 0.523 \\
\hline P \% & 0.67 & 0.83 & 0.75 & 0.75 & 0.065 \\
\hline K \% & 1.18 & 1.62 & 1.4 & 1.4 & 0.179 \\
\hline Ca ppm & 4871 & 4575 & 3607 & 4351 & 539.79 \\
\hline Mg ppm & 8000 & 7145 & 8675 & 7940 & 626.06 \\
\hline Fe ppm & 11.31 & 15.21 & 12.78 & 13.1 & 1.608 \\
\hline Zn ppm & 115.2 & 130 & 122.3 & 122.5 & 6.044 \\
\hline Cu ppm & ND & ND & ND & ND & \\
\hline
\end{tabular}

* ND $=$ No detectado

puede afectar la dinámica de los elementos esenciales para el desarrollo y crecimiento de los cultivos, sin embargo, se sabe que la acelga es un cultivo tolerante a las sales (Shannon et al., 2000). De acuerdo a Abad et al. (2001) el rango óptimo para el pH es de 5.3-6.5 y para la conductividad eléctrica el valor óptimo es menor a $500 \mu \mathrm{S} / \mathrm{cm}$, por lo que en referencia a estos valores el obtenido en el sustrato estudiado es alto. Paredes et al. (2005), produjeron un compost con la adición de residuos de la producción de aceite de oliva el cual resultó con alta conductividad eléctrica y en el que no obtuvieron un efecto negativo en las plantas de acelga.

Rendimiento de acelga en sustrato orgánico y efecto de B. subtilis y T. harzianum

Al analizar los datos de tres cortes de biomasa fresca de la acelga (Tabla 3), nos muestran que el primer corte realizado no presentó diferencias estadísticas significativas (Tukey ( $a=0.05)$, sin embargo, el tratamiento testigo presentó el valor más alto, lo que hace notar que el sustrato es adecuado para el desarrollo de la acelga; y el valor menor en producción de biomasa fue para el tratamiento Th_C2 (T. harzianum). Sin embargo, al evaluar los resultados del segundo corte, el tratamiento con la cepa CH90_3T presentó el mayor rendimiento, con un $27 \%$ mayor en biomasa fresca que el testigo, los demás tratamientos $\mathrm{A}_{1}$ Th_C2, $\mathrm{A}_{1}+\mathrm{Th} \_\mathrm{C} 2$, $\mathrm{A}+\mathrm{CH} 90 \_3 \mathrm{~T}, \mathrm{CH} 90 \_3 \mathrm{~T}+\mathrm{Th} \_\mathrm{C} 2, \mathrm{CH} 90 \_3 \mathrm{~T}+\mathrm{Th} \_\mathrm{C} 2+\mathrm{A} 1$ presentaron menor rendimiento en biomasa fresca en relación al testigo. Por otra parte, la combinación de las dos cepas de $B$. subtilis (tratamiento $\mathrm{A} 1+\mathrm{CH} 90 \_3 \mathrm{~T}$ ) presentó un $38 \%$ menos rendimiento que el testigo. Los valores del tercer corte fueron de $750.1 \mathrm{~g}$ de peso fresco obtenidos al inocular la cepa CH90_3T, mientras que para la combinación de CH90_3T + Th_C2 el peso fresco fue sólo de $220.46 \mathrm{~g}$. Los valores totales muestran que la cepa CH90_3T y A1 tuvieron un efecto favorable sobre la biomasa fresca de acelga en el sustrato orgánico. El uso de consorcios microbianos para el crecimiento de las plantas, han sido descritos en diversas investigaciones mostrando el efecto sinérgico entre microorganismos benéficos (Kumar et al., 2012; Walker et al., 2012). Sin embargo, para el desarrollo de consorcios microbianos que estimulan el crecimiento de las plantas, se sugiere que previo a la evaluación de crecimiento, se realicen pruebas de interacción dentro de condiciones in vitro de los microorganismos (Choure y Dubey, 2012). En este trabajo se observó el efecto negativo sobre el rendimiento en la biomasa fresca del cultivo de acelga con la inoculación combinada de las cepas $\mathrm{A} 1+\mathrm{CH} 90 \_3 \mathrm{~T}$ que pudiera deberse a un posible antagonismo entre dichas cepas. El efecto negativo en el rendimiento de biomasa fue observado también con el uso de la cepa Th_C2 de $T$. harzianum sola o en combinación con $B$. subtilis y de la combinación de cepas de $B$. subtilis. Resultados similares se han reportado y en la que se muestra una clara dependencia en el modo de inoculación utilizado al combinar organismos biocontroladores (Abeysinghe, 2009).

Tabla 3. Evaluación del efecto de la inoculación de B. subtilis ( $\mathrm{CH} 90$ y $\left.\mathrm{A}_{1}\right)$ y T. harzianum (Th_C2) sobre la biomasa fresca de acelga ( $\mathrm{g} /$ contenedor) en sustrato orgánico, con un análisis estadístico de medias Tukey $(\mathrm{a}=0.05)$.

Table 3. Evaluation of the effect of the inoculation of B. subtilis (CH90 and A1) and T. harzianum (Th_C2) on fresh Swiss chard biomass ( $\mathrm{g} /$ container) in organic substrate, with a Tukey statistical means analysis (a $=0.05$ ).

\begin{tabular}{lcccc}
\hline Tratamiento & Primer Corte & Segundo Corte & Tercer Corte & Total \\
\hline CH90 & $258.21 \pm 38.31 \mathrm{a}$ & $349.26 \pm 27.12 \mathrm{a}$ & $150.02 \pm 64.86 \mathrm{a}$ & $757.5 \pm 51.08 \mathrm{a}$ \\
$\mathrm{A} 1$ & $279.67 \pm 58.41 \mathrm{a}$ & $261.06 \pm 88.19 \mathrm{ab}$ & $127.78 \pm 85.10 \mathrm{ab}$ & $668.51 \pm 143.09 \mathrm{ab}$ \\
Th_C2 & $237.25 \pm 41.49 \mathrm{a}$ & $249.22 \pm 91.18 \mathrm{ab}$ & $104.6 \pm 52.85 \mathrm{ab}$ & $591.07 \pm 102.01 \mathrm{abc}$ \\
$\mathrm{A} 1+$ Th_C2 & $274.2 \pm 26.57 \mathrm{a}$ & $227.76 \pm 22.71 \mathrm{ab}$ & $60.89 \pm 10.88 \mathrm{ab}$ & $562.85 \pm 33.99 \mathrm{bc}$ \\
$\mathrm{A} 1+\mathrm{CH} 90$ & $267.83 \pm 35.82 \mathrm{a}$ & $168.55 \pm 53.01 \mathrm{~b}$ & $50.59 \pm 18.66 \mathrm{~b}$ & $486.98 \pm 62.63 \mathrm{c}$ \\
CH90+Th_C2 & $244.05 \pm 12.11 \mathrm{a}$ & $189.41 \pm 57.83 \mathrm{~b}$ & $44.09 \pm 38.01 \mathrm{~b}$ & $477.55 \pm 81.97 \mathrm{c}$ \\
CH90+Th_C2+A1 & $289.96 \pm 22.12 \mathrm{a}$ & $187.14 \pm 27.07 \mathrm{~b}$ & $59.55 \pm 12.06 \mathrm{ab}$ & $536.66 \pm 12.33 \mathrm{bc}$ \\
Control & $296.92 \pm 53.89 \mathrm{a}$ & $274.97 \pm 77.05 \mathrm{ab}$ & $89.59 \pm 35.05 \mathrm{ab}$ & $661.48 \pm 104.61 \mathrm{ab}$ \\
\hline
\end{tabular}

Prueba de comparación de medias Tukey ( $\mathrm{a}=0.05)$. Las medias con letras iguales por columna no son estadísticamente diferentes. 
Con respecto a los valores sobre la biomasa seca de acelga (Tabla 4), el efecto de los tratamientos fueron notables en el primer corte, mostrando que la adición de las combinaciones de las cepas A1 + Th_C2, A1 + CH90_3T y CH90_3T + Th_C2 y CH90_3T + Th_C2+A y el tratamiento A1 mostraron diferencias estadísticas significativas (Tabla 4). Para el segundo corte, el peso seco se vio favorecido con los tratamientos CH90_3T, A, Th_C2, A + Th_C2 y no difirieron del testigo. Para el tercer corte, ningún tratamiento mostró diferencias estadísticas significativas. En producción orgánica con uso de sustratos únicos, el agotamiento de nutrimentos puede ocurrir, y uno de los elementos que debe ser suministrado de manera continua, ya sea de manera orgánica o convencional con fertilizantes, es el nitrógeno por ser un elemento lábil y requerido para diversos procesos en la planta (Marshner, 2012; Barker y Mills, 1980).
La acelga es un suministro de nutrientes al consumidor, y resulta importante mantener los niveles adecuados durante su desarrollo. Un aspecto importante de esta investigación, fue evaluar la capacidad de los microorganismos en favorecer la absorción nutrimental. Los resultados de peso fresco y peso seco se ven complementados con el análisis foliar, donde las cepas de $B$. subtilis (CH90_3T y A1) influyeron significativamente en la absorción de $\mathrm{K}, \mathrm{Ca}, \mathrm{Mg}, \mathrm{Fe}, \mathrm{Mn}$ y Zn (Tabla 5). El efecto de la cepa de T. harzianum (Th_C2), aunque no tuvo un efecto significativo en las variables de crecimiento, contribuyó a la mayor absorción de $\mathrm{K}$, Ca y $\mathrm{Cu}$ comparado con el testigo. Las cepas de $B$. subtilis $\left(\mathrm{A}_{1}\right.$ y CH90_3T) favorecieron la absorción de más del $100 \%$ del Zn respecto al testigo; este efecto en parte es responsable de los mayores rendimientos y mayor crecimiento de las plantas, ya que el $\mathrm{Zn}$ es un elemento que tiene una gran influencia sobre

Tabla 4. Evaluación del efecto de la inoculación de $B$. subtilis (CH90_3T y $\left.A_{1}\right)$ y T. harzianum (Th_C2) sobre la biomasa seca de acelga ( $\mathrm{g} /$ contenedor) en sustrato orgánico, con un análisis estadístico de medias Tukey $(a=0.05)$.

Table 4. Evaluation of the effect of the inoculation of B. subtilis (CH90_3T and A1) and T. harzianum (Th_C2) on the dry biomass of chard (g/container) in organic substrate, with a statistical analysis of Tukey means $(a=0.05)$.

\begin{tabular}{lcccc}
\hline Tratamiento & Primer Corte & Segundo Corte & Tercer Corte & Total \\
\hline CH90_3T & $75.59 \pm 2.24 \mathrm{bc}$ & $134.86 \pm 2.09 \mathrm{a}$ & $79.78 \pm 6.90 \mathrm{a}$ & $290.24 \pm 5.30 \mathrm{a}$ \\
\hline $\mathrm{A}_{1}$ & $96.27 \pm 4.02 \mathrm{ab}$ & $102.91 \pm 6.95 \mathrm{ab}$ & $71.58 \pm 9.53 \mathrm{a}$ & $270.76 \pm 13.88 \mathrm{ab}$ \\
\hline TH2 & $55.69 \pm 1.95 \mathrm{~cd}$ & $110.28 \pm 8.07 \mathrm{ab}$ & $65.67 \pm 6.64 \mathrm{a}$ & $231.65 \pm 12.57 \mathrm{ab}$ \\
\hline $\mathrm{A}_{1}+\mathrm{TH} 2$ & $100.09 \pm 1.94 \mathrm{a}$ & $88.61 \pm 1.77 \mathrm{ab}$ & $38.63 \pm 1.38 \mathrm{a}$ & $227.34 \pm 2.77 \mathrm{ab}$ \\
\hline $\mathrm{A}_{1}+\mathrm{CH} 90 \_3 \mathrm{~T}$ & $101.54 \pm 2.72 \mathrm{a}$ & $78.10 \pm 4.91 \mathrm{~b}$ & $31.29 \pm 2.31 \mathrm{a}$ & $210.94 \pm 6.30 \mathrm{ab}$ \\
\hline CH90_3T+TH & $86.87 \pm 0.86 \mathrm{ab}$ & $75.77 \pm 4.63 \mathrm{~b}$ & $27.27 \pm 4.70 \mathrm{a}$ & $189.92 \pm 7.64 \mathrm{~b}$ \\
\hline CH90_3T+TH+A & $89.91 \pm 1.37 \mathrm{ab}$ & $79.46 \pm 2.30 \mathrm{~b}$ & $30.09 \pm 1.22 \mathrm{a}$ & $199.47 \pm 1.41 \mathrm{~b}$ \\
\hline Control & $51.37 \pm 1.86 \mathrm{~d}$ & $114.00 \pm 6.39 \mathrm{ab}$ & $49.36 \pm 3.86 \mathrm{a}$ & $214.73 \pm 9.37 \mathrm{ab}$ \\
\hline
\end{tabular}

Prueba de comparación de medias Tukey $(a=0.05)$. Las medias con letras iguales por columna no son estadísticamente diferentes.

Tabla 5. Análisis foliar de la acelga.

Table 5. Foliar analysis of Swiss chard.

\begin{tabular}{|c|c|c|c|c|c|c|c|c|c|}
\hline \multirow[t]{2}{*}{ Tratamiento } & $\mathbf{N}$ & $\mathbf{P}$ & $\mathbf{K}$ & $\mathrm{Ca}$ & Mg & $\mathrm{Fe}$ & $\mathrm{Cu}$ & Mn & Zn \\
\hline & \multicolumn{3}{|c|}{$\%$} & \multicolumn{6}{|c|}{ Ppm } \\
\hline CH90_3T & 4.30 & 0.78 & 1.71 & 300.1 & 1462 & 122 & 8 & 90 & 800 \\
\hline$A_{1}$ & 4.00 & 1.11 & 2.55 & 316.1 & 1657 & 29 & 9 & 71 & 880 \\
\hline Th_C2 & 4.10 & 0.95 & 0.77 & 131.3 & 179 & 12 & 24 & 55 & 377 \\
\hline $\mathrm{A}_{1}+\mathrm{Th} \_\mathrm{C} 2$ & 3.50 & 1.04 & 2.07 & 281.9 & 1513 & 102 & 5 & 54 & 685 \\
\hline $\mathrm{A}_{1}+\mathrm{CH}$ 90_3T & 4.30 & 1.11 & 0.99 & 95.5 & 171 & 10 & 1 & 23 & 195 \\
\hline CH90_3T + Th_C2 & 4.60 & 1.10 & 0.25 & 76.1 & 155 & 29 & 0 & 31 & 235 \\
\hline $\mathrm{A}_{1}+\mathrm{CH} 90 \_3 \mathrm{~T}+\mathrm{Th} \_\mathrm{C} 2$ & 4.60 & 0.97 & 0.30 & 96.45 & 165 & 33 & 7 & 53 & 302 \\
\hline Control & 4.10 & 0.92 & 0.31 & 91.05 & 160 & 15 & 8 & 41 & 390 \\
\hline
\end{tabular}

Final del formulario

Arrastra y suelta el archivo o enlace aquí para traducir el documento o la página web.

Arrastra y suelta el enlace aquí para traducir la página web.

El tipo de archivo que has soltado no es compatible. Inténtalo con otro tipo de archivo.

El tipo de enlace que has soltado no es compatible. Inténtalo con otro tipo de enlace. 
la síntesis de la hormona del crecimiento Ácido Indol Acético (Mengel y Kirkby, 2001), el Ca en la síntesis de la pared celular y el Mg en la fotosíntesis que se reflejó en la mayor producción de biomasa seca en los tratamientos de CH90_3T y A1. La necesidad de recuperar $\mathrm{N}$ en el sustrato, fue necesario evidenciarlo por la reducción del rendimiento durante el segundo y tercer corte, así como el área foliar y los cambios en coloración asociados con deficiencias de N (Barickmana y Kopsell, 2016) como el amarillamiento observado (Datos no presentados).

El efecto de los tratamientos y del compost sólo, se vio reflejado en la producción de biomasa de la acelga. Pues la acelga como cultivo de hojas, es apreciada por su alto contenido de clorofila, pigmentos carotenoides y otros nutrientes como vitaminas A, C y D (Reif et al., 2013), que pueden ser observados por el consumidor en su color verde obscuro. La acelga además aporta minerales como calcio, hierro, fósforo, fibra y proteína (Maynard y Hochmuth, 1997). Además, la calidad puede ser afectada por el manejo aunque se ha reportado que el contenido nutrimental puede ser similar en manejo convencional y orgánico (Moreira, et al. 2003), no así el impacto ecológico que puede ser diferente y en el manejo de cultivos con el uso de promotores del desarrollo puede ser apreciado.

Otros de los elementos absorbidos en mayores proporciones respecto al testigo, fueron el potasio (más del 800 $\%$ ), calcio (más del $300 \%$ y magnesio (más del $1000 \%$ ); la respuesta lógica, contemplando las funciones fisiológicas de estos elementos esenciales, es el vigor, el crecimiento y la resistencia a insectos y microorganismos patógenos, que impacta en la calidad nutritiva de las hojas comestibles de esta especie.

Por otro lado, el hongo T. harzianum influyó negativamente sobre el crecimiento de las plantas, sobre todo cuando se asoció con las cepas de la bacteria B. subtilis (Tabla 3); además, la asociación del hongo con las dos cepas de la bacteria, propició las condiciones para que las plantas absorbieran en general menores cantidades de los elementos esenciales respecto al testigo y a las cepas por separado (Tabla 2).

A pesar de los beneficios de utilizar inoculantes microbianos y de sustratos orgánicos de desechos agroindustriales, la aplicación en cultivos de interés agrícola es poco común, debido a la problemática de llevarlo a producción a escalas mayores (Nelson, 2004). Sin embargo, para garantizar la seguridad alimentaria es necesario contar con las prácticas tradicionales, como los huertos familiares. En dichos huertos la inoculación y el uso de sustratos orgánicos podrían garantizar la producción de alimentos para el consumo familiar, asegurando cultivos inocuos, la disminución de fertilizantes orgánicos, etc.

\section{CONCLUSIONES}

La introducción de microorganismos benéficos son un claro ejemplo de los efectos positivos para las prácticas agrícolas sustentables. El hongo T. harzianum tuvo una influencia positiva sobre la producción y absorción nutri- mental respecto al testigo (sustrato); sin embargo, las cepas CH90_3T y $A_{1}$ de la bacteria $B$. subtilis tuvieron una influencia significativa sobre la producción tanto de materia fresca como de materia seca y la absorción de elementos esenciales para el crecimiento de la acelga respecto al testigo y al hongo T. harzianum. Este trabajo resalta la importancia de utilizar materiales considerados como desechos agroindustriales por su contenido nutrimental y microorganismos que promuevan el crecimiento de las plantas para una agricultura sustentable.

\section{AGRADECIMIENTOS}

Este trabajo fue desarrollado gracias al financiamiento del proyecto SIP 20161000 del Instituto Politécnico Nacional.

\section{REFERENCIAS}

Abad, M., P. Noguera y S. Burés. 2001. National inventory of organic wastes for use as growing media for ornamental potted plant production: case study in Spain. Bioresource Technology. 77: 197-200.

Abedini Aboksari H., Hashemabadi D. y Kaviani, B. 2018. Application of biofertilizer for Pelargonium peltatum growth in new organic substrates. Journal of Plant Nutrition. 41: 137-149.

Abeysinghe, S. 2009. Effect of Combined Use of Bacillus subtilis CA32 and Trichoderma harzianum RU01 on Biological Control of Rhizoctonia solani on Solanum melongena and Capsicum annuum. Plant Pathology Journal. 8: 9-16.

Adesemoye, A.O. y Kloepper, J.W. 2009. Plant-microbes interactions in enhanced fertilizer-use efficiency. Appl Microbiol Biotechnol. 85:1.

Anthony H., Mark G.y Margot L. 1992. The new Royal Horticultural Society, dictionary of gardening (4). London: The Macmillan Press and The Stockton Press.

Barickmana, T.C. y Kopsell, D.A. 2016. Nitrogen form and ratio impact Swiss chard (Beta vulgaris subsp. cicla) shoot tissue carotenoid and chlorophyll concentrations. Scientia Horticulturae. 204: 99-105.

Barker, A.V. y Mills, H.A. 1980. Ammonium and nitrate nutrition of horticultural crops. Hortic Rev. 2 394-424.

Boldrin A., Andersen J.K., Moller J., Christensen T.H. y Favoino E. 2009. Composting and compost utilization: accounting of greenhouse gases and global warming contributions. 27: 693-693.

Choure K. y R.C. Dubey. 2012. Development of Plant Growth Promoting Microbial Consortium Based on Interaction Studies to Reduce Wilt Incidence in Cajanus cajan L. Var. Manak. World Journal of Agricultural Sciences. 8: 118-128.

Chu, H.Y., Y. Hoden y K. Yagi. 2004. Nitrogen oxide emissions and soil microbial activities in a Japanese andisol as affected by N-Fertilizer management. Soil Sci Plant Nutr. 50: 287-292.

Kumar V., Dhall P., Kumar R., Prakash Singh Y. y Kumar A. 2012. Bioremediation of Agro-Based Pulp Mill Effluent by Microbial Consortium Comprising Autochthonous Bacteria. The Scientific World Journal. 2012: 1-7.

Lampkin, N. 1990. Organic farming (pp. 557-575). Ipswich: Farming Press.

Lugtenberg B. y Kamilova F. 2009. Plant-growth-promoting rhizobacteria. Annu Rev Microbiol. 63: 541-556.

Volumen XXI, Número 3 
Marschner, H., 2012. Mineral Nutrients of Higher Plants, 3rd ed. Academic Press,New York, NY.

Maynard, D.N. y Hochmuth, G.J. 1997. Knott's Handbook for Vegetable Growers, $4^{\text {th }}$ ed. John Wiley and Sons, New York, NY.

Medeiros de C.D., de Sousa F.K.C., de Sousa V.F., Barros dos A.R.S., Duarte B.R., Cavalcante N.J.G., de Sousa N.G.H., Alves F.H. 2008. Qualidade de mudas de alface em função de substratos com e sem biofertilizante. Hortic Bras. 26: 186189.

Mengel K. y Kirkby E.A. 2001. Principles of plant nutrition. 849 pp. 5th edn. Dordrecht: Kluwer Academic Publishers.

Moreira, M.R., Roura, S.I. y del Valle, C.E. 2003. Quality of Swiss chard produced by conventional and organic methods. LWT - Food Science and Technology. 36: 135-141.

Nelson L.M. 2004. Plant growth promoting rhizobacteria (PGPR): Prospects for new inoculants. Crop Manage. 3.

Olsen, S. R., y Sommers. L.E. 1982. Determination of available phosphorus. En "Method of Soil Analysis", vol. 2, ed. A. L. Page, R. H. Miller, y D. R. Keeney, 403. Madison, Wl: American Society of Agronomy.

Kleifeld O. y Chet I. 1992. Trichoderma harzianum-interaction with plants and effect on growth response. Plant and Soil. 144: 267-272.

Pan I., Dam B. y Sen S. K. 2012. Composting of common organic wastes using microbial inoculants. 3 Biotech. 2: 127-134.

Paredes C., Cegarra J. Bernal M.P.y Roig A. 2005. Influence of olive mill wastewater in composting and impact of the compost on a Swiss chard crop and soil properties. Environment International. 31: 305-312.

Pérez-García A., Romero D. y de Vicente A. 2011. Plant protection and growth stimulation by microorganisms: biotechnological applications of Bacilli in agriculture. Current Opinion in Biotechnology. 22: 187-193.
Pinos-Rodríguez J.M., García-López J.C., Peña-Avelino L.Y., Rendón-Huerta J.A., González-González C. y Tristán-Patiño F. 2012. Impactos y regulaciones ambientales del estiércol generado por los sistemas ganaderos de algunos países de América. Agrociencia. 46: 359-370.

Pyo, Y.H., Lee, T.C., Longedra, L., Rosen, R.T. 2004. Antioxidant activity and phenolic compounds of Swiss chard (Beta vulgaris subspecies cycla) oleoresins. Food Chemistry. 85: 19-26.

Reif, C., Arrigoni, E., Scharer, H., Nystrom, L. y Hurrell, R.F. 2013. Carotenoid database of commonly eaten Swiss vegetables and their estimated contribution to carotenoid intake. J Food Compos Anal. 29: 64-72.

Rivera-Cruz M.C., Trujillo Narcía A., Córdova Ballona G., Kohler J., Caravaca F. y Roldán A. 2008. Poultry manure and banana waste are effective biofertilizer carriers for promoting plant growth and soil sustainability in banana crops. 40: 30923095.

Shannon M.C., Grieve C.M., Lesch S.M. y Draper J.H. 2000. Analysis of Salt Tolerance in Nine Leafy Vegetables Irrigated with Saline Drainage Water. JASHS 125(5) 658-664.

Souza R., Ambrosini A. y Passaglia L.M.P. 2015. Plant growthpromoting bacteria as inoculants in agricultural soils. Genet Mol Biol 38: 401-419.

Walker, V., Couillerot, O., Von Felten, A., Bellvert, F., Jansa, J., Maurhofer, M., Bally, R., Moenne-Loccoz, Y., y Comte, G. 2012. Variation of secondary metabolite levels in maize seedling roots induced by inoculation with Azospirillum, Pseudomonas and Glomus consortium under field conditions. Plant and Soil. 356: 151-163. 